\title{
Optimization and Design of RPL Routing Protocol for 6LowPAN Wireless Sensor Network
}

\author{
Baoguo Zeng \\ Chengdu Vocational \& Technical College of Industry, Chengdu 610218, China \\ 505958148@qq.com
}

Keywords: 6LowPAN; RPL routing protocol; fuzzy logic theory

\begin{abstract}
LowPAN (IPv6 over Low-power wireless Personal Area Networks) is a low-speed wireless personal area network standard based on IPv6, the key technology of which is IPv6 Routing Protocol for Low-Power and Lossy Networks. In a 6LowPAN network, the network topology is typically dynamically formed, so the RPL routing protocol must be able to discover the connections and select neighbor nodes to establish and maintain the network topology. Aiming at the problem that RPL routing decision can only use single routing metric, fuzzy logic theory is used to introduce multiple routing metrics in routing decision, the objective function is designed and implemented, and the optimal parent node is selected by using the objective function to optimize RPL routing protocol.
\end{abstract}

\section{Introduction}

With the rise of Internet of Things technology and the arrival of large data age, Wireless Sensor Network (WSN) technology has been widely used and rapidly developed. The traditional IP network is extended to the wireless sensor network, which realizes the interconnection of WSN and the Internet. It is the research and the application hot spot of the wireless sensor network. As the traditional IP technology is not suitable for the resource-constrained wireless sensor networks, the IETF (Internet Engineering Task Force) formed a 6LowPAN working group to solve the problem of IPv6JIS technology applied to WSN. After analyzing the existing wireless sensor network routing protocols, the working group proposed the RPL (Routing Protocol for Low-Power and Lossy Networks) routing protocol for low-power lossy network applications ${ }^{[1]}$. RPL is a distance vector routing protocol based on IPv6. The topology network based on directed acyclic graph is constructed by using Metric and Objective Function (OF). Because the objective function defined by IETF only depends on the single route metric to optimize the path, there are some problems such as inefficient routing and not meeting the application requirements. For example, when we choose the lower embedded technology extension (ETX) path, the real-time performance of the path may not meet the requirements. Therefore, the objective function cannot provide the optimal route with a single routing metric. The aim of this study is to introduce multiple routing metrics in routing decision-making by fuzzy logic theory to design and implement the objective function, and use Cooja simulation software to simulate and analyze the fuzzy optimization routing performance ${ }^{[2-3]}$.

\section{Improved algorithm of RPL Routing Protocol Based on 6LowPAN}

RPL is an IPv6-based distance vector routing protocol designed for low-power lossy networks. It is based on the topology concept of Directed Acyclic Graph (DAG). It constructs Destination Oriented Directed Acyclic Graph (DODAG) by using objective function (OF) and metric (Metric) set ${ }^{[4]}$. Through the above method, the objective function can use the set of metrics and constraints to calculate the optimal path for each RPL node to reach the root node (Sink or LBR) ${ }^{[5]}$.

The algorithm used in this paper is the objective function based on fuzzy logic, called OF-FL (The Objective Function Based on Fuzzy Logic). Fuzzy logic can abstract reasoning for the values from any range, and the algebraic operation of the imprecise information can be completed by it rigorously. At 
the same time, fuzzy logic can combine multi-objective problem with expert system, and the complexity of fuzzy logic algorithm is low.

In order to establish a real-time, reliable and energy-efficient routing algorithm, the fuzzy logic method is adopted, and the routing metric of nodes and links reflected by delay time, hop number, ETX and node energy is considered in the fuzzy logic system. The trapezoidal membership function is widely used. We choose the trapezoidal membership function for each of the four metrics, and the language variable for the routing metric has three values. The membership of each value is calculated by the membership function.

Actually, fuzzy reasoning comprehensively evaluates a group of input values based on fuzzy inference rules, resulting in a determined output range, that is, fuzzy output ${ }^{[6]}$. After the fuzzy output is de-fuzzified, the fuzzy logic system finally outputs the exact value of 0 to 100 , which represents the quality of the neighbor nodes, and then selects the node with the highest quality as the best parent node. The value of fuzzy output defined in OF-FL is: \{Excellent, very good, good, low good, bad, low bad, awful\}.

OF-FL uses Mamdani-type fuzzy reasoning and the implication operator:

$R_{C}: R_{C}=A \rightarrow B=A \times B$

$\mathrm{A}$ is the fuzzy set of inference antecedent, $\mathrm{B}$ is the consequent of the fuzzy rule, $R_{C}$ is the fuzzy relationship.

The "max-min" operation is used to compose the fuzzy relation. After the operation rules are defined, if the input is $A^{\prime}$, then we can get the formula (2) after input $B^{\prime}$ :

$B^{\prime}=A^{\prime}$ o $R_{C}=A^{\prime}(A \times B)$

0 is the "max-min" operation.

The fuzzy output of fuzzy reasoning uses the centroid de-russification method:

$Q=\frac{\sum_{i=1}^{N} W_{i} \times \mu_{A}\left(W_{i}\right)}{\sum_{i=1}^{N} \mu_{A}\left(W_{i}\right)}$

$W_{i}$ is the corresponding domain value of the rule $i, N$ is the number of rules triggered in fuzzy reasoning, $\mu_{A}\left(W_{i}\right)$ is the membership value corresponding to the domain value.

\section{Performance evaluation and comparative analysis of the improved algorithm}

\subsection{Simulation parameter setting}

We use the Cooja simulation software to simulate the RPL routing protocol performance based on OF-FL ${ }^{[7]}$. This simulation experiment uses Tmote Sky node to set up 100 router nodes and 1 edge router node, the latter is the root node of DODAG. All nodes are arranged in the area of $600 \mathrm{~m} \times 600 \mathrm{~m}$, using wireless channel 26 , and each node is working in the strongest emission state. The RPL router node sends a datagram every 60 seconds to the root node after 60 seconds from the start of the simulation to build a stable topology. The simulation time is set as at least 60 minutes to ensure that each round of simulation can be aggregated in a stable state.

\subsection{Simulation results}

We compared, analyzed and evaluated the RPL routing protocol based on OF-FL, the RPL routing protocol based on OF0 and the RPL routing protocol based on MRHOF (ETX) (the ETX is regarded as the routing metric) from the following five aspects:

Average Hop Count: It is the hop count between the RPL router node and the DODAG root node.

End-to-end delay: It is the time interval between RPL router nodes transmitting data to the node that received the response signal sent by the DODAG root node.

Packet loss ratio: It is the ratio of the number of packets received by the root node to the total number of packets output by the router node. 
Average residual energy: It is used to assess the survival time of the network, and is the average of all nodes to save energy.

Average number of changes of parent nodes: It is the number of changes of the parent nodes in the network, and used to evaluate the stability of the network topology. Excessive number of changes in the parent node will result in instability of the network topology, but will improve the quality of the path and the performance of the route ${ }^{[8]}$.

(1) The average number of hops

Figure 1 shows the simulation results of the average number of hops of the routing protocol based on OF0, MRHOF and OF-FL.

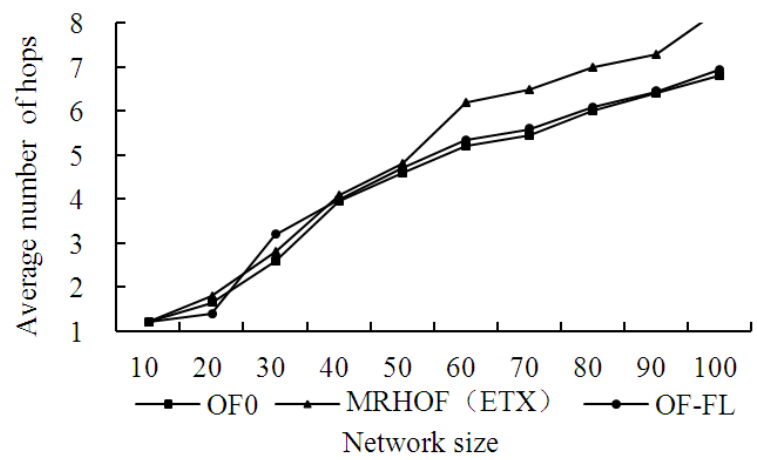

Figure 1 the average number of hop

It can be seen from the figure 1, in the sparse network that the number of nodes of the router is less than 50, the performance difference among the three objective functions is very small. This is because in a sparse network, the neighbor nodes of a node are fewer, resulting in a small number of father nodes that can be selected by the node. However, compared to MRHOF (ETX), the average number of hops corresponding to OF-FL is smaller in a dense network with more than 50 router nodes. When the number of router nodes is more than 80 , OF0 and OF-FL are closer to the average number of hops that correspond to each other, which proves that OF-FL can reduce the number of hops of the router in DODAG.

(2) End-to-end delay

Figure 2 shows the simulation results of the average end-to-end delay based on the number of hops to the root node of the RFL routing protocol with OF0, MRHOH (ETX) and OF-FL as objective functions, respectively. It can be seen from Figure 2 that the average end-to-end delay time of the three objective functions is less than 4 seconds. Although OF0 is aimed at reducing the number of hops, the average end-to-end delay time corresponding to OF0 is higher than that of MRHOF and OF-FL. This further confirms that the shortest path corresponding to the delay is not necessarily the smallest. Because in the routing process, some of the nodes in the shortest path will be congested, resulting in the increase of delay. In addition, when the number of hops arriving at the root node is high (from 6 hop), the average end-to-end delay of OF-FL is significantly less than that of OF0 and MRHOF (ETX). Therefore, the average end-to-end delay of OF-FL is less than that of other existing single-route metric objective functions.

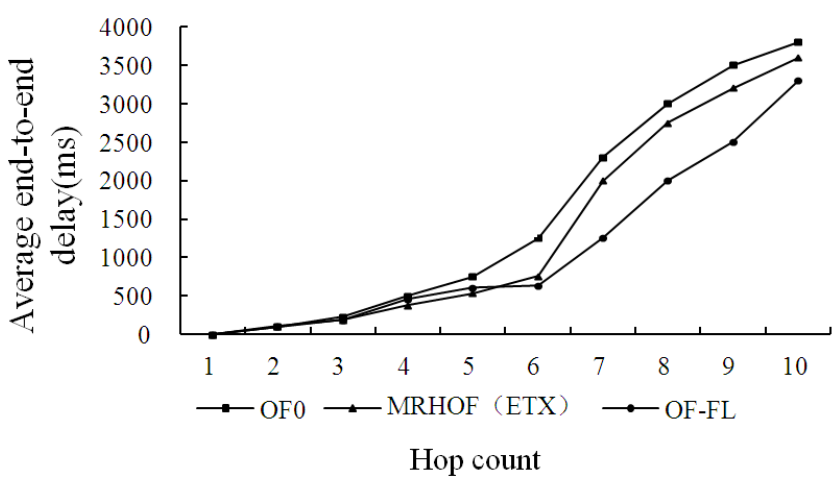

Figure 2 Average end-to-end delay time 
(3) Packet loss rate

The corresponding packet loss rate of OF0, MRHOF (ETX) and OF-FL are compared to evaluate the reliability of the network corresponding to OF-FL. The simulation results are shown in Figure 3. Figure (a) is the simulation results when the throughput is 1 pkts/min, and Figure (b) is the simulation results when the throughput is $6 \mathrm{pkts} / \mathrm{min}$.

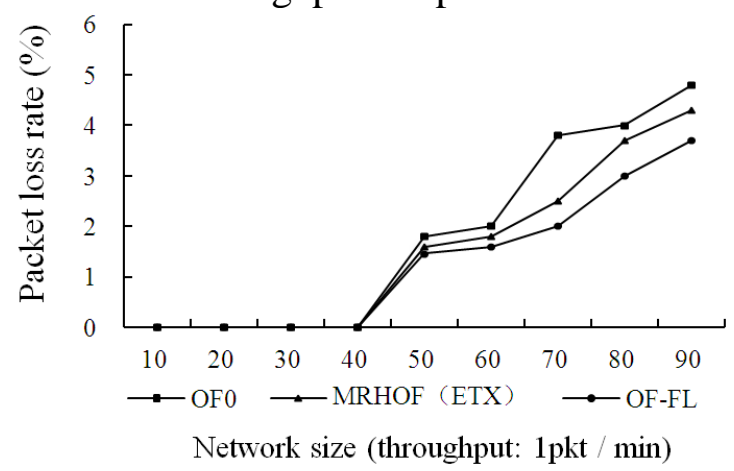

(a)

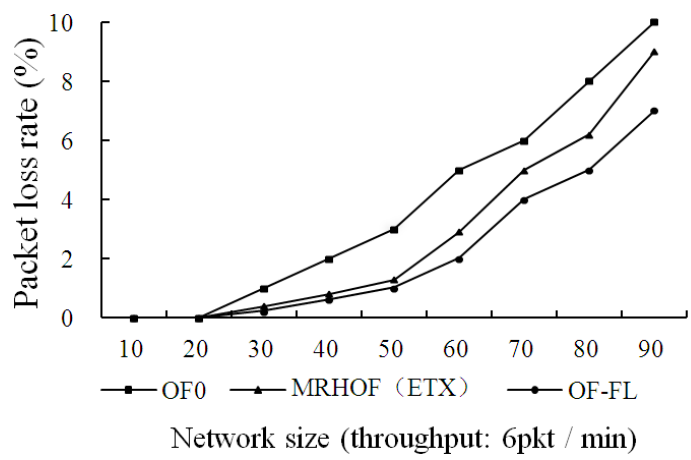

(b)

Figure 3 Loss rate of packet

As can be seen from the figure, in a sparse network with few nodes, the packet loss rate is very low. As the network density increases, the packet loss rate becomes more and more serious. From Figure (a) we can know that, compared with OF0, the packet loss rate of OF-FL and MRHOF (ETX) is low. This is because that the OF0 aims at minimizing the Rank value of the parent node and can't provide a high-quality link. At the same time, the OF0 is prone to congestion at the selected parent node, so OF0 corresponds to a higher packet loss rate. OF-FL and MRHOF (ETX) packet loss rate is roughly the same, because both regard the ETX as the routing metric. When choose the parent node, they minimize the link ETX, so both the packet loss rate is low. Compared the graph (a) with the graph (b), we can see that the packet loss rate in the network increases significantly when the throughput of the node increases (6 pkts / min). This is because the larger the throughput will lead to network congestion and packet collision phenomenon. In graph (b), the packet loss rate of OF-FL is significantly lower than that of OF0 and MRHOF (ETX). This is because OF-FL allows discarding a small number of packets. So, in the high throughput network, OF-FL performance even better than MRHOF (ETX).

(4) The average residual energy

The residual energy of the router node is used to evaluate the lifetime of the network. The most important factor affecting the network lifetime is the balance of the node energy consumption. The simulation results are shown in Figure 4.

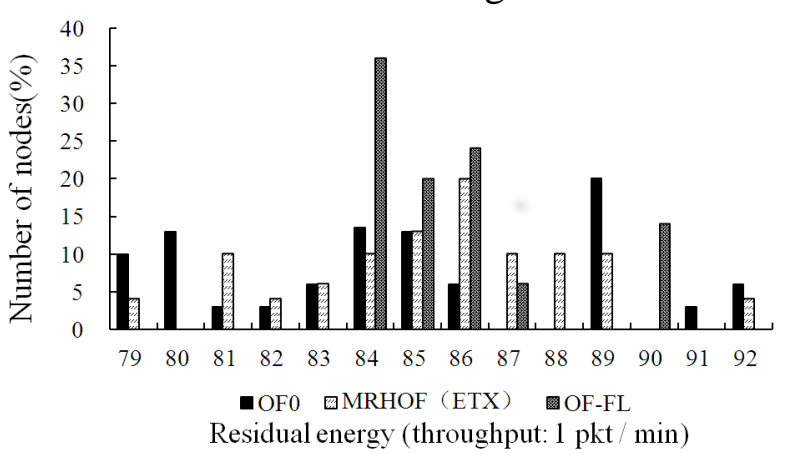

(a)

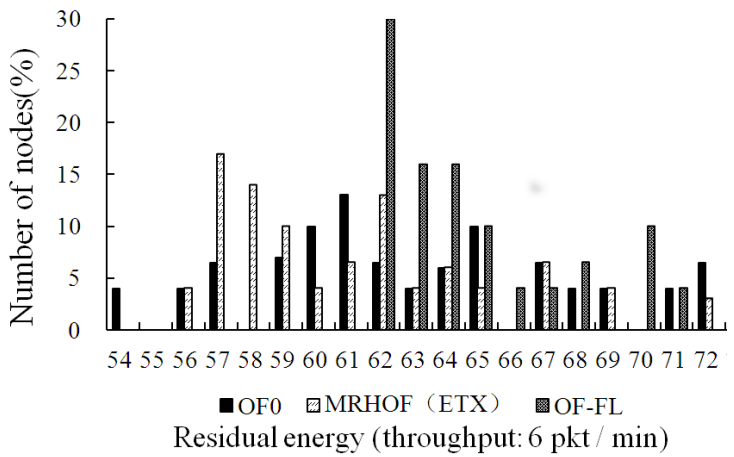

(b)

Figure 4 Residual energy

From Figure 4, we can see that the residual energy distribution of the nodes corresponding to OF0 and MRHOF (ETX) are not even. From Figure (a), when the throughput is 1pkts / min, in the stimulation experiment that OF0 corresponds, the residual energy of $23 \%$ nodes is less than $80 \%$, the residual energy of $39 \%$ nodes is between $81 \%$ and $85 \%$, the residual energy of $26 \%$ nodes is $86 \%$ and $90 \%$, and the residual energy of $12 \%$ nodes is above $91 \%$. So, the residual energy of some nodes will be used up earlier, and the network's survival time will be reduced. In the simulation of OF-FL in Figure (a), the node energy consumption is more balanced ( $81 \%$ of the node's residual energy is below 
86\%). Compared the graph (a) with the graph (b), we can see that the energy consumption of the nodes in the network will be faster when the throughput is $6 \mathrm{pkts} / \mathrm{min}$. The OF-FL with the MRHOF both have low residual energy nodes at the two throughputs.

(5) The average numbers of changes of parent nodes

The stability of the network topology is evaluated by the average number of changes of the parent nodes. The simulation results are shown in Figure 5. It can be seen from the figure that the average change times of the parent nodes of $\mathrm{OF} 0$ is 0.2 times per hour, and the average change times of parent nodes of MRHOF (ETX) is 0.252 times per hour, and the average parent nodes change frequency is very low. This is because OF0 and MRHOF (ETX) target only aim at the minimum Rank value, but do not optimize the selection process of the parent node. The average change number of parent-node in OF-FL is 0.275 times per hour, slightly higher than OF0 and MRHOF (ETX).

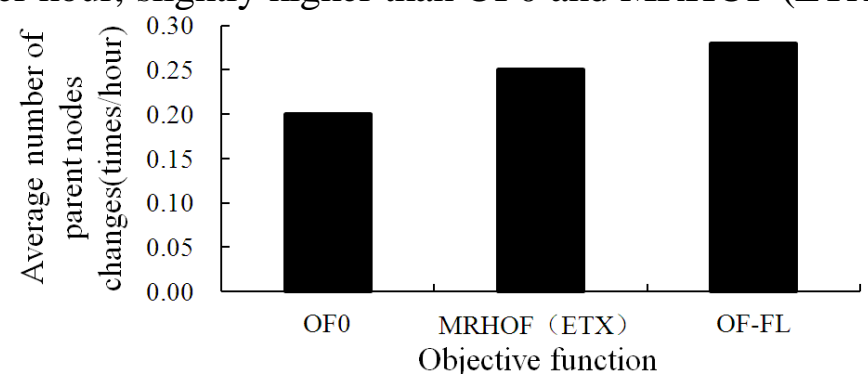

Figure 5 The number of changes in the parent node

\section{Conclusion}

The RPL routing decision-making uses only a single routing metric for 6LowPAN wireless sensor network. The fuzzy logic theory is used to introduce a variety of routing metrics into the routing decision-making. The OF-FL objective function is designed and implemented. The quality of each node is obtained by the fuzzy logic system to get the best parent node. OF-FL can delay the appearance of the energy depletion node. The higher change times can improve the quality of the path and routing performance in OF-FL network.

\section{References}

[1].Kalyoncu, S. Wireless solutions and authentication mechanisms for contiki based internet of things networks. School of Information Science Computer \& Electrical Engineering, 2014.

[2].Bouaziz M, Rachedi A. A survey on mobility management protocols in Wireless Sensor Networks based on 6LoWPAN technology [J]. Computer Communications, 2014, 99(1):1-16.

[3] Sherburne M, Marchany R, Tront J. Implementing moving target IPv6 defense to secure 6LoWPAN in the internet of things and smart grid[C]// Cyber and Information Security Research Conference. ACM, 2014:37-40.

[4] Deru L, Dawans S, Ocaña M, et al. Redundant Border Routers for Mission-Critical 6LoWPAN Networks [J]. 2014, 281:195-203.

[5] Lohith Y S, Narasimman T S, Anand S V R, et al. Link Peek: A Link Outage Resilient IP Packet Forwarding Mechanism for 6LoWPAN/RPL Based Low-Power and Lossy Networks (LLNs) [C]// IEEE International Conference on Mobile Services. IEEE, 2015:65-72.

[6] Rghioui A, Khannous A, Bouhorma M. Denial-of-Service attacks on 6LoWPAN-RPL networks: Issues and practical solutions [J]. Journal of Advanced Computer Science \& Technology, 2014, 3(2): 143.

[7] Khelifi N, Oteafy S, Hassanein H, et al. Proactive maintenance in RPL for 6LowPAN[C]// Iwcmc. 2015:993-999.

[8] Hellaoui H, Koudil M. Bird Flocking Congestion Control for CoAP/RPL/6LoWPAN Networks[C]// the Workshop on Iot Challenges in Mobile \& Industrial Systems. 2015:25-30. 